\title{
OPINIÃO E REVISÃO
}

\section{Complicações pós-operatórias das anastomoses colorretais}

\author{
Post-surgical complications of the colorectal anastomosis
}

\author{
JÚLIO CÉSAR MONTEIRO SANTOS JR ${ }^{1}$ \\ ${ }^{1}$ Titular da Sociedade Brasileira de Coloproctologia.
}

SANTOS JR JCM. Complicações pós-operatórias das anastomoses colorretais. Rev bras Coloproct, 2011;31(1): 98-106.

\begin{abstract}
RESUMO: As complicações da terapêutica das doenças colorretais que envolvem ressecções e anastomoses não são raras e, frequentemente, são graves. Possuem extensões mórbidas variáveis que prolongam o período de internação hospitalar, aumentam substancialmente o custo do tratamento, perpetuam sequelas funcionais e contribuem para indesejável índice de mortalidade. As mais temíveis complicações são: a infecção e a deiscência da anastomose. Esta quase sempre é a causa mais nefasta da infecção e se expressa como infecção profunda. A deiscência da anastomose tem definição, incidência, etiologia e fatores de riscos como alvos de controvertidas opiniões, o que torna difícil a elaboração de esquemas profiláticos e planejamentos terapêuticos universais. Sua consequência mais grave é a peritonite generalizada que culmina com a sepse. As complicações menores, menos frequentes, são: hemorragia anastomótica, estenose e fístula. Aquela, às vezes, tem solução espontânea, mas pode eventualmente necessitar de procedimentos terapêuticos agressivos; a estenose, em geral associada a fatores que determinam a deiscência tais como a isquemia e a imperfeição técnica, na maioria das vezes, pode ser tratada de forma conservadora, mesmo que intervencionista, com o uso de dilatadores rígidos ou plásticos. Outras vezes, no entanto, pode exigir a abordagem cirúrgica, com a desvantagem do aumento da morbidade, principalmente nos casos em que o segmento precisa ser ressecado e a anastomose refeita. A última, com gravidade variável, é, no entanto, alvo de manipulação conservadora, mas às vezes envolve meios complexos de terapêutica com resultados que nem sempre são coroados de sucesso. No presente manuscrito, pretende-se dar uma visão geral dessas complicações, suas causas, evoluções, diagnósticos e tratamentos.
\end{abstract}

Palavras-chave: anastomose cirúrgica; fístula anastomótica; hemorragia gastrointestinal; fístula intestinal.

\section{INTRODUÇÃO}

As complicações que se associam e decorrem das intervenções cirúrgicas sobre o intestino grosso são várias e envolvem conotações sistêmicas ou regionais. Algumas têm relações indiretas com o ato operatório, e outras são consequências diretas das técnicas e táticas empregadas, razão porque quase todas elas têm início no intraoperatório ${ }^{1}$. Dentro do contexto de complicações pós-operatórias, é possível citar: as insuficiências cardiopulmonares (respiratórias e cardíacas - dependentes ou não); as do trato urinário - infecciosas ou não; as vasculares periféricas, incluindo o tromboembolismo; e as que afetam a ferida cirúrgica, superficial ou profunda, na maioria das vezes associadas às infecções e que podem ser causa ou resultado da deiscência da anastomose.

Esses eventos pós-operatórios diferem na incidência, mas todos são relevantes, porque aumentam a morbidade, efetivam a maior permanência hospitalar, aumentam os custos do tratamento, podem ser fatais e, quando a complicação é a deiscência da anastomose em pacientes operados de câncer do reto, os prognósticos das operações curativas pioram, pois esta complicação favorece a recidiva local ${ }^{2-5}$.

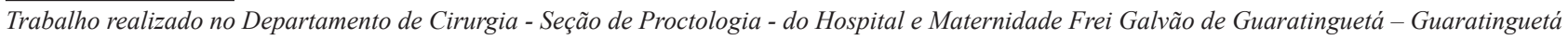
(SP), Brasil.

Recebido em: 21/12/2010

Aprovado em: 14/04/2011 
A anastomose merece atenção especial, não só por sua mais temível complicação, que é a deiscência, que, associada a infecções locorregionais, pode ser devastadora, como por outros elementos que incluem sua incidência variável com dependência de aspectos anatômicos, os métodos de investigação, a definição e os fatores de riscos $6,7,8,9,10,11$. Além da deiscência, outras três complicações que despertam interesses são as fístulas ou os sínus - na realidade, dois aspectos da deiscência - o sangramento e a estenose. Essas complicações podem ser agrupadas em precoces e tardias. A deiscência e a hemorragia seriam precoces; a estenose, tardia, mas em algumas circunstâncias as deiscências, como fístula ou como sínus, podem ser, pelo menos do ponto de vista de manifestação clínica e de diagnóstico, consideradas tanto precoce, na forma de tardias. Neste último caso, muitas vezes esse tipo de complicação implica com a estenose.

A exata frequência da deiscência não é conhecida, mas a ocorrência não é incomum, fato que, aliado à gravidade decorrente do escape do conteúdo intestinal para a cavidade peritoneal, impõe medidas, sobretudo as consideradas preventivas, além daquelas que seriam capazes de identificá-la precocemente para a pronta definição do tratamento.

A identificação precoce, com imediato tratamento, fatores essenciais na antecipação da evolução catastrófica da deiscência, preconizada por Goligher et al. ${ }^{12,13}$, pode ser obtida por meio de exame radiológico contrastado (enema com gastrografina), feito no pós-operatório imediato. Esses mesmos autores fizeram a demonstração precoce da deiscência num estudo que envolveu 135 pacientes submetidos a ressecções colorretais, por carcinoma ou doença diverticular ( 84 com anastomoses altas e 51 com anastomoses baixas) $)^{12,13}$. Neste estudo, foram identificadas 65 deiscências (35\%), sendo $8 \%$ com expressão clínica e $27 \%$, apenas radiológica.

Pesquisa semelhante feita pelo autor deste artigo $^{14}$, em 117 pacientes consecutivos, teve os seguintes resultados: foram identificadas 25 deiscências anastomóticas (21,3\%), sendo 17 (14,5\%) com significado exclusivamente radiológico e $8(6,8 \%)$ com manifestação clínica, dentre as quais três pacientes $(37,5 \%)$ foram reoperados.
Neste manuscrito, as mencionadas complicações das anastomoses executadas no intestino grosso serão analisadas.

\section{COMPLICAÇÕES PRECOCES}

\section{Deiscência da anastomose \\ Aspectos clínicos e fatores de riscos}

A constatação da deiscência anastomótica e sua qualificação foram motivos para classificá-la em gru$\operatorname{pos}^{1}$ : subclínico (expressão exclusivamente radiológica de importância prática desprezível) e clínico. No grupo clínico, reuniu-se as seguintes formas clínicas: com sinais de sepse em variados graus; com peritonite localizada; com peritonite localizada e fístula fecal externa e com peritonite generalizada.

Vale destacar que esta classificação pode ser usada para orientar o tratamento da deiscência anastomótica $^{1}$. Outro aspecto importante, além do reconhecimento da incidência e variação da expressão clínica, prende-se aos fatores de riscos causais que envolvem as técnicas e os tipos das anastomoses; os tipos se relacionam com o local onde são confeccionadas. No conjunto de dados, devem ser distinguidos os três seguintes elementos que favorecem a deiscência: primeiro, a técnica inapropriada devido à aproximação das partes sob tensão; a má irrigação das partes a serem anastomosadas; e a hemorragia, que, com eventuais aspectos agravantes, são dependentes do cirurgião ${ }^{10}$. Segundo, são os fatores relacionados aos elementos preexistentes, em geral associados ao paciente e à doença, tais como a infecção ou contaminação locorregional, à desnutrição e ao edema ${ }^{15,16}$, entre outros ${ }^{11,17,18}$, e, terceiro, quanto aos tipos de anastomoses, situação em que os maiores riscos de deiscências envolvem o segmento retal do intestino grosso e dependem da proximidade da anastomose com o canal ${ }^{10,14,19-23}$. Enquadram-se, também, nesse grupo, as anastomoses íleo-anais, com bolsa ileal, cujos índices de deiscências anastomóticas variam de 5 a $10 \%{ }^{24,25}$ e têm como fator agravante o paciente com história de retocolite ulcerativa, usando dose de prednisona maior que $40 \mathrm{mg}$, por dia ${ }^{15}$.

Os outros fatores de risco que podem ter significado preditivo em relação à deiscência e não devem ser desprezados são: diabetes, alcoolismo crônico, obesidade, uso de drenos e duração do ato operatório ${ }^{8,9,18,22,26-31}$. 


\section{Profilaxia e diagnóstico precoce}

Esses conhecimentos propiciam a oportunidade de medidas preventivas, bem como prepara o cirurgião para o diagnóstico mais precoce. As mais valorosas medidas preventivas, passíveis de domínio, são as que se relacionam com a técnica da confecção da anastomose ${ }^{1}$. Quaisquer procedimentos que eventualmente venham a ser associados às anastomoses têm poder de somar morbidades, mas não de reduzir o índice de deiscência. Contudo, considerando-se os altos riscos das situações que envolvem anastomoses baixas, os pacientes do sexo masculino, os diabéticos, os fumantes ou os alcoólatras, aqueles submetidos à terapia neoadjuvante e a operações de longa duração, a profilaxia recomendável é a derivação intestinal proximal, indicada, nestes casos, para atenuar as consequências da deiscência ${ }^{4,8,10,15,18,16,32,33}$.

O diagnóstico precoce depende, fundamentalmente, de dois fatores: primeiro, a definição pré-operatória das condições de risco e, segundo, a participação incisiva do cirurgião por um alto grau de suspeição.

As manifestações clínicas no pós-operatório imediato e os auxílios inquestionáveis para o diagnóstico instantâneo podem ser precoces ou tardias. As precoces ocorrem num paciente afebril e já bem disposto, dentro das primeiras 36 ou 48 horas depois da operação, são decorrentes do escape que ocorre por causa de defeito técnico na confecção da anastomose. Em geral, fazem-se notar pela aceleração do pulso e a extemporânea passagem de conteúdo intestinal pelo ânus, acompanhados de palidez cutânea, desânimo, inapetência e a insistente volta ou permanência no leito.

Os exames laboratoriais, difíceis de serem interpretados nesse instante, são de pouco valor, pois se misturam com as alterações relativas às reações metabólicas, ao trauma, às provocadas pela infecção emergente. $\mathrm{O}$ exame direto da linha anastomótica nem sempre é conclusivo e, antes que as manifestações clínicas se exacerbem, o melhor método de imagem, mesmo quando há a disponibilidade para a tomografia ${ }^{34}$, é o exame radiológico contrastado feito com o contraste iodado, solúvel em água ${ }^{12,14,34}$.

As tardias podem ser de dois tipos: as primeiras, cujas ocorrências de fato se estabelecem após o quarto dia do pós-operatório imediato (entre o quarto e oitavo dia), podem ter, logo no início, manifestações clínicas exuberantes, nas quais a gravidade tem muita relação com o nível das anastomoses - são mais graves nas anastomoses confeccionadas acima da reflexão peritoneal do que nas mais baixas - e, as segundas, que são descobertas no final do período do pós-operatório precoce (até o $30^{\circ}$ dia) ou as que são diagnosticadas no pós-operatório tardio, com exames de imagem - ultrassonográfico ou tomográfico, subsidiados pelo quadro clínico e achado de exame físico.

Nas tardias do pós-operatório imediato, em que os diagnósticos são exclusivamente clínicos, o momento mais catastrófico do quadro, para um paciente que evoluía bem nos dois primeiros dias após a operação, é precedido por alterações insidiosas, as quais, muitas vezes, escapam da avaliação crítica do cirurgião e que são: taquisfigmia, taquipneia, apatia, inapetência, palidez cutânea, leucocitose e febre. Em geral, os movimentos intestinais, que já estavam presentes, cessam. A existência desses dados, fortemente prenunciadores da sepse, força a mais racional das associações, ou seja, estabelece o vínculo entre infecção e deiscência anastomótica. Por isso, é mais seguro considerar o estado geral do paciente nas primeiras horas que sucedem o ato operatório e estabelecer que, para cada período seguinte, independente do sexo e da idade, o paciente deve estar clinicamente melhor, incluindo dados objetivos e subjetivos da avaliação.

\section{Tratamento}

O tratamento da deiscência anastomótica pode ser inicialmente expectante ou cirúrgico, na dependência do bloqueio ou livre vazamento do conteúdo intestinal para a cavidade abdominal.

Nas circunstâncias em que houver deiscência e as reações locais forem suficientemente eficazes para limitar o escape com bloqueio biológico, resultando no "abscesso" localizado, a oportunidade de tratamento inicial é expectante seguido da facilitação de drenagem externa, por via percutânea guiada por exames de imagens ou, eventualmente, pela própria anastomose, se esta for facilmente acessível, como acontece para aquelas confeccionadas no reto médio e distal. Nesses casos, os cuidados adjuvantes e subsequentes incluem a limpeza mecânica da loja e a antibioticoterapia, para alvo polimicrobiano. Este procedimento resultará numa fístula cuja evolução poderá ser subsequentemente tratada com a derivação intestinal proxi- 
mal, planejada e executada de acordo com criteriosa avaliação.

Quando há vazamento para a cavidade abdominal, ocorrências mais comuns nas retossigmoidectomias anteriores com reconstrução acima da reflexão peritoneal ou em outros segmentos dos cólons; o quadro clínico - de início insidioso e muitas vezes considerado irrelevante - é caracterizado muito mais por alterações sistêmicas do que locais, já que a peritonite no pós-operatório imediato, em geral, não cursa com as mesmas características sintomatológicas observadas nas peritonites que evoluíram como consequência de foco abdominal primário (peritonite secundária a uma afecção abdominal primária).

No pós-operatório, além das lesões causadas pela agressão cirúrgica, há as alterações orgânicas reacionais neuroendócrinas e metabólicas, com significativo efeito de supressão imune, o que confere ao período pós-cirúrgico o caráter de anergia, inclusive no meio celular ${ }^{35-37}$, ao qual soma-se a infecção. O expoente mais grave dessa manifestação inicial, favorecida por fatores conjunturais, é a sepse devido ao foco peritoneal, cujo curso envolve respostas pró- e antiinflamatórias ${ }^{38}$. Os sinais de alarme iniciais, mesmo na ausência de dor abdominal, são a taquicardia, a taquipneia, a febre e a leucopenia (abaixo de 4.000 leucócitos) ou a leucocitose (acima de 12.000) ${ }^{39}$ e devem ser vistos como exigência para medidas terapêuticas emergenciais, que incluem a agressiva abordagem do foco infeccioso, com suporte médico intensivo ${ }^{40} \mathrm{e}$ antibioticoterapia, na qual associam-se dois ou mais antibióticos ${ }^{41,42}$. Nessas condições, o tratamento cirúrgico deve ser imediato, com amplo acesso à cavidade peritoneal, para eliminar a fonte de infecção, reduzir o contaminante peritoneal, evitar a peritonite continuada e, eventualmente, proceder com a derivação intestinal proximal ${ }^{41,42}$.

A antecipação dessas medidas se baseia, portanto, no alto grau de suspeição, no reconhecimento dos riscos inerentes ao procedimento cirúrgico que gerou a complicação e nos fatores coadjuvantes que favorecem a deiscência da anastomose ${ }^{21,33,19}$. No entanto, a tendência da maioria dos cirurgiões é ser relutante em aceitar que o paciente possa ter esse tipo de complicação, sobretudo, eles evitam considerar que as complicações do pós-operatório podem ter bases no intraoperatório ${ }^{1}$. Assim, racionalizam e negam o fato, perdendo a oportunidade do tratamento precoce ${ }^{43}$, fato que contribui para o insucesso do tratamento ${ }^{44-47}$. A prevenção das expressões sistêmicas dos distúrbios enunciados é o passo mais seguro para atingir os melhores resultados e bons prognósticos, oferecendo a oportunidade para o exame contrastado precoce, com contraste iodado solúvel em água ${ }^{43}$, o que, sem dúvida, ajuda planejar o tratamento cirúrgico.

O cirurgião deve reconhecer que, na peritonite pós-operatória, a intervenção cirúrgica com o objetivo terapêutico é a segunda lesão mencionada nos postulados de Bone ${ }^{38,39,48}$, razão para que essa segunda lesão seja, tanto quanto possível, limitada e efetiva em seus propósitos $^{49}$, dadas as graves consequências do trauma, em si, e da possibilidade de recorrência ou persistência da peritonite ${ }^{50}$. Por isso, recomenda-se que a intervenção cirúrgica na vigência da peritonite seja feita para resolver o problema e evitar as laparotomias programadas $^{50}$.

O planejamento cirúrgico, precedido pela antibioticoterapia combinada, é definido no intraoperatório, obedecidos os fundamentos estabelecidos para o tratamento da peritonite difusa grave, sobretudo limitando ao máximo o ato na satisfação do primeiro objetivo, que é eliminar a fonte de contaminação, sem a aventura de maiores descolamentos cirúrgi$\cos ^{50}$, de tal forma que o fechamento ou reparo da lesão, exclusão e, eventualmente, ressecção limitada do foco da infecção associados à limpeza mecânica com lavagem exaustiva da cavidade são os procedi-

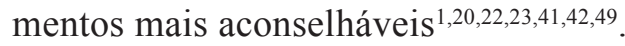

A anastomose, cujo escape provocou a peritonite difusa, pode ser reparada e protegida com uma derivação proximal ou desfeita e, neste caso, as extremidades, se possível, exteriorizadas. O segmento distal pode ser colocado num dos extremos da incisão cirúrgica, como fístula mucosa, e o proximal convenientemente arranjado como uma colostomia terminal. Se o segmento distal não alcançar a pele, procede-se com seu fechamento, identificação e fixação num local mais próximo possível da colostomia, de modo que possa ser facilmente encontrado, facilitando a reconstrução do trânsito, quando for oportuno. Essa tática, em geral, se aplica às situações em que o diagnóstico não foi precoce, mas conduzido pelas manifestações sistêmicas da sepse. Cabe aqui, não fugindo do escopo desta seção, salientar que a avaliação e condução 
do paciente com deiscência anastomótica implicam no conhecimento das bases fisiopatológicas da peritonite pós-operatória difusa grave, porque ela, muito mais do que a deiscência em si - nessas circunstâncias, apenas um detalhe - deve ser o alvo principal do processo terapêutico, já que a taxa de mortalidade nessas peritonites varia de 22 a $55 \%$ e tem como fatores determinantes o retardo no tratamento e a maior dificuldade de controle da sepse peritoneal ${ }^{51}$.

No grupo de 96 pacientes com peritonite pósoperatória de várias etiologias, estudados por Mulier et al. ${ }^{51}$, em 2003, houve 29 óbitos (30\%). Neste, os autores denominaram a terapêutica como precoce, quando o tempo decorrido entre os primeiros sinais e sintomas clínicos de infecção foram definidos com um ou mais dos seguintes sintomas, ou seja, dor anormal, febre axilar $\geq 38,5^{\circ} \mathrm{C}$, hipoxemia $\left(\mathrm{PaO}_{2}<65 \mathrm{mmHg}\right)$, pressão sistólica menor que $100 \mathrm{mmHg}$, ou oliguria (débito urinário menor que $500 \mathrm{~mL} /$ dia), e a terapêutica cirúrgica foi menor que 24 horas. Definiu-se como terapêutica retardada quando o tempo decorrido entre o aparecimento dos primeiros sinais e sintomas mencionados e a terapêutica cirúrgica foi maior que 24 horas. No método de estudo, os autores destacaram vários fatores implicados com a gravidade da situação, dentre os quais, destacamse os seguintes: compartimento de localização da fonte de infecção (abdômen superior ou inferior); natureza da fonte de contaminação (gástrica, biliar e intestinal); natureza do exsudato peritoneal (purulento, biliar e fecal); tratamento dado a fonte de infecção (fechamento da deiscência, reconstrução da anastomose, ressecção e derivação e apenas drenagem).

As doenças que motivaram a operação eletiva foram colorretal $(39,6 \%)$, gastroduodenal $(14,6 \%)$, biliar $(11,6 \%)$, intestinal $(10,4 \%)$, vascular $(7,3 \%)$, hepática $(4 \%)$, urológica $(3 \%)$, ginecológica $(2 \%)$ e transplante de rim (2\%). As causas das peritonites foram: deiscência de anastomose $(58,3 \%)$, lesões iatrogênicas (lacerações) $(16,7 \%)$, perfuração de divertículo (diverticulite) $(15,6 \%)$, entre outros. O fluido peritoneal estava purulento (19,8\%), bilioso (23\%) e fecal $(57,3 \%)$. No esquema de tratamento, a relaparotomia planejada a cada 48 horas foi feita, sendo $24(46,3 \%)$ dos 55 pacientes com peritonite fecal e $5(12 \%)$ de 41 pacientes com peritonite purulenta ou biliar.

A mortalidade foi de 30\% (29 pacientes) - 24 $(82,7 \%)$ deles morreram na Unidade de Terapia Inten- siva - em oito pacientes, a fonte da sepse não pôde ser controlada; em sete, a peritonite difusa não pôde ser controlada; seis morreram de choque séptico a despeito do controle local e peritoneal, oito pacientes morreram de infecção localizadas em órgãos distantes, principalmente, por complicações pulmonares.

Deu-se destaque para quatro fatores independentes relacionados com a morte, tais como: incapacidade para controlar a fonte de infecção, incapacidade para controlar a sepse peritoneal, idade e confusão mental. Neste estudo, não houve influência direta da terapêutica precoce comparada com a retardada; porém, o número de horas de uma e de outra não foi significativamente diferente. No entanto, quanto mais longo foi o tempo entre o aparecimento dos primeiros sinais e sintomas, maior foi a dificuldade para o controle da sepse peritoneal.

As deiscências de anastomoses do intestino delgado, anastomoses íleocólicas ou das bolsas ileais, em situações favoráveis, podem ser reparadas ou refeitas e colocadas imediatamente abaixo da linha de sutura da parede abdominal, com o propósito de orientar uma fístula enterocutânea, se o reparo não for bemsucedido $^{15}$.

\section{FÍSTULA}

As fístulas anastomóticas, dependendo da sua topografia e da variada gravidade, constituem-se numa das principais causas de morbimortalidade nas operações de reconstrução das vísceras ocas. Nas operações coloproctológicas, as deiscências de anastomoses podem resultar em comunicação com a pele (fístula cólon cutânea), vagina e trato geniturinário masculino ${ }^{15}$. Dependendo da localização do orifício de saída, a fístula bem formada tem resolução espontânea. Assim, a abordagem inicial desse tipo de complicação deve ser sempre expectante, com controle da infecção e cuidados para providenciar a adequada coleção do efluente da fístula, principalmente, com proteção para a pele. Grande parte dessas fístulas está associada ao abscesso intra-abdominal, que deve ser convenientemente drenado. O período de tempo com a conduta expectante deve ser prolongado para mais de oito semanas, pelo menos para que estejam minimizadas as reações fibróticas e inflamatórias intraperitoneais, o que tornaria um eventual procedimento cirúrgico subsequen- 
te menos perigoso com maior favorecimento para um bom resultado no tratamento da fístula ${ }^{52}$.

Para a maior parte das fístulas cólon-cutâneas, as recomendações são as seguintes ${ }^{53}$ :

- tratamento conservador - dieta alimentar suspensa ou com baixo teor de resíduos e antibióticos;

- considere, se houver possibilidade, tentar selar o orifício com adesivo de fibrina ${ }^{54-56}$;

- $\quad$ se o tratamento conservador for ineficaz, indicase a operação cirúrgica;

- $\quad$ se o paciente necessitar de procedimento cirúrgico, a técnica é fundamental e deve ser escolhida para evitar a recorrência. A secção do segmento envolvido e a reconstrução de uma nova anastomose são as escolhas de padrão-ouro;

- outra opção é a excisão da fístula, fechamento e fistulectomia - procedimentos reservados para os pacientes com doenças associadas significativas e nas situações em que há muitas aderências, tornando a mobilização do segmento envolvido difícil ou perigosa.

\section{HEMORRAGIA NA ANASTOMOSE}

A passagem de sangue escuro nas primeiras evacuações, expressão de sangramento ocorrido logo após a confecção das anastomoses, é evento comum ${ }^{15}$, mas as hemorragias originadas naqueles sítios e que exigem medidas intervencionistas são raras, variam em menos de 1 até $3 \% 0^{43,57,58}$ e são mais frequentes naquelas feitas com os grampeadores ${ }^{15}$. Na maioria das vezes, cessam espontaneamente, às vezes exigem reposição volêmica ou até procedimentos locais pela via endoscópica com cauterização, uso de clipe ou injeção local de vasoconstritores ${ }^{57-60}$. Raramente necessitam de uma intervenção maior, como a relaparotomia $^{58}$.

\section{Complicação tardia}

\section{Estenose-etiologia e incidência}

A estenose de uma anastomose tem relação muito próxima com eventos etiológicos que não são independentes: a isquemia, a deiscência e a técnica ${ }^{61,62,63}$.

A isquemia poderia estar implicada com a estenose, supondo que o processo inflamatório consequente e a fibroplasia subsequente reuniriam os elementos necessários para a reparação com retração e estreita- mento circunferencial durante o processo de cicatrização. Contudo, na vigência da isquemia, precedendo as primeiras reações biológicas da cicatrização, a ocorrência comum seria a deiscência da anastomose, do tipo subclínico, cujo curso inicial foi inexpressivo, mas concorrente para excessiva fibrose na fase tardia da cicatrização. Por outro lado, estima-se que para que a estenose seja significativa, a deiscência tem que envolver 25 a $50 \%$, isto é um quarto da metade da circunferência da anastomose $\mathrm{e}^{1,43}$.

A incidência da estenose é relativamente alta, variando de 3 a $30 \%{ }^{64,65}$ e é mais comum nas anastomoses mecânicas do que nas confeccionadas manualmente (2:1), como pode ser observado num escrutínio feito entre membros da Sociedade Americana de Cirurgiões Colorretais; $76 \%$ estavam localizadas no reto e sigmoide; $70(57 \%)$, no reto e $23(18,7 \%)$, no sigmoide, num grupo de 123 pacientes com estenose ${ }^{65}$.

Os fatores pré-operatórios identificados como facilitadores da estenose foram: obesidade (23\%), abscessos (10\%), deiscência da anastomose (12\%), infecção pélvica $(10,5 \%)$ e irradiação pós-operatória $(5,7 \%)^{65}$.

Em 53\% dos pacientes, a estenose foi tratada e resolvida com dilatação, mas 34 pacientes (28\%) precisaram de intervenção abdominal cirúrgica ${ }^{65}$.

\section{Tratamento}

As estenoses, em operações cirúrgicas sobre o intestino grosso, ocorrem principalmente nas anastomoses colorretais, em geral, envolvendo as confeccionadas no reto distal, portanto, de fácil acesso. Estes são fatores determinantes para a escolha do procedimento terapêutico que, comumente, é conservador, orientado pelas manifestações clínicas do estreitamento e a eventuais dificuldades funcionais decorrentes, não só relativas à exoneração das fezes, mas também associadas à incontinência intestinal. Assim, após o exame físico e a avaliação do diâmetro do anel anastomótico, a conduta pode ser orientada para o amolecimento das fezes, para o uso de enemas ou de supositórios ${ }^{43}$, seguidos ou não de dilatação digital ou com dilatadores metálicos de Hegar, os quais podem ser usados para anastomoses um pouco mais altas, para as quais, podemos, também, empregar os dilatadores curvos tipo Benique ${ }^{66}$. Os dilatadores endoscópicos com balões de variados diâmetros são 
outros dispositivos para o tratamento conservador das estenoses colorretais, seguros e seguidos de bons resultados ${ }^{67}$.

As estenoses de anastomoses colorretais podem, eventualmente, precisar de um método mais agressivo do que as simples dilatações. Nesses casos, impõese a ampliação cirúrgica que, na maioria das vezes, é feita por via anal. $\mathrm{O}$ anel estenótico é seccionado no sentido longitudinal e fechado no transversal. Nas es- tenoses colorretais mais altas, o procedimento pode, bem-sucedido, ser feito por via endoscópica, usando técnica de microcirurgia com os dispositivos usados nas papilotomias endoscópicas ${ }^{68}$. Todavia, o tratamento da estenose, associado ao maior índice de morbidade, num número não-desprezível de pacientes, pode exigir a abordagem abdominal, circunstância em que o segmento estenótico deve ser ressecado e uma nova anastomose, construída ${ }^{65}$.

\begin{abstract}
Complications of colorectal diseases management that involve resections and anastomosis are not rare; they are frequently severe. With morbid variable extensions, they extend the period of hospital, considerablyincrease the treatment cost, perpetuate functional sequels, and contribute to undesirable high rate mortality. The most frightening complication is the anastomosis dehiscence, whose definition, incidence, etiology, and factors of risks are targets of controversial opinions, causing difficulty to prepare preventive universal schemes. The most severe consequence of the anastomosis leakage is the generalized peritonitis, culminating in the sepsis that is the primary cause of surgically related mortality. The least and less frequent complications are the anastomotic hemorrhage, stenosis, and fistula. The anastomotic hemorrhage, fistula and stenosis most times, can be treated as a nonsurgical form. This manuscript was intended by to provide a general view of these complications, their causes, evolutions, diagnoses, and treatments.
\end{abstract}

Keywords: anastomosis,surgical; anastomotic leak; gastrointestinal hemorrhage; intestinal fistula.

\section{REFERÊNCIAS}

1. Moossa AR, Block JE. Complications of Colorectal Operations. In: Operative Colorectal Surgery. 1st ed. Block\&Moossa Ed, Philadelphia. W.B. Saunders Company 1994; p. 507-18.

2. Bell SW, Walker KG, Richard MJ, Sinclair G, Dent OF, Chapuis PH, et al. Anastomotic leakage after curative anterior resection results in a higher prevalence of local recurrence. Br J Surg 2003;90(10):1261-8.

3. Law WL, Choi HK, Lee YM, Ho JW, Seto CL. Anastomotic leakage is associated with poor long-term outcome in patients after curative colorectal resection for malignancy. J Gastrointest Surg 2007;11(1):8-15.

4. McArdle CS, McMillan DC, Hole DJ. Impact of anastomotic leakage on long-term survival of patients undergoing curative resection for colorectal cancer. Br J Surg 2005; 92(9):1150-4.

5. Walker KG, Bell SW, Richard MJFX, Mehanna D. Anastomotic Leakage is predictive of diminished survival after potentially curative resection for colorectal cancer. Ann Surg 2004;240(2):255-9.

6. Hyman NH. Managing anastomotic leaks from intestinal anastomoses. Surgeon 2009;7(1):31-5.

7. Bruce J, Krukowski ZH, Al-Khairy G, Russell EM, Park KG. Systematic review of the definition and measurement of anastomotic leak after gastrointestinal surgery. Br J Surg 2001;88(9):1157-68.

8. Alberts JC, Parvaiz A, Moran BJ. Predicting risk and diminishing the consequences of anastomotic dehiscence following rectal resection. Colorectal Dis 2003;5(5):478-82.
9. Barbuscia M, Gorgone S, Rizzo AG, Punturieri L, Sanò M, De Luca M, et al. Anastomotic dehiscence in colorectal surgery. G Chir 2002;23(8-9):310-4.

10. Cong ZJ, Fu CG, Wang HT, Liu LJ, Zhang W, Wang H. Influencing factors of symptomatic anastomotic leakage after anterior resection of the rectum for cancer. World J Surg 2009;33(6):1292-7.

11. Alves A, Panis Y, Trancart D, Regimbeau JM, Pocard M, Valleur P. Factors associated with clinically significant anastomotic leakage after large bowel resection: multivariate analysis of 707 patients. World J Surg 2002;26(4):499-502.

12. Goligher JC, Grahan NG, de Dombal FT. Anastomotic dehiscence after anterior resection of the rectum and sigmoid. Br J Surg 1970;57:109-18.

13. Goligher JC, Simpkins KC, Lintott DJ. A controlled comparison of one- and two-layer techniques of suture for high and low colorectal anastomoses. Br J Surg 1977;64:609-14.

14. Martins Jr A, Aprilli F, Guimarães AS, Santos Jr JCM, Rocha JJR. Leakages of colorectal anastomosis. Dig Dis Sci 1986;31:443.

15. Dietz DW, Bailey HR. Postopertive complications. In: Bruce GW, James WF, et al. The ASCRS Textbook of Colon and Rectal Surgery, 1st ed. New York: Springer Science\&Business Media, LLC; 2007. p. 141-53.

16. Wolff BG, Fleshman JW, Beck DE, Pemberton JH, Wexner SD, editors. The ASCRS Textbook of Colon and Rectal Surgery. 1st ed. New York: Springer Science\&Business Media, LLC; 2007.

17. Choi HK, Law WL, Ho JW. Leakage after resection and intraperitoneal anastomosis for colorectal malignancy: 
analysis of risk factors. Dis Colon Rectum 2006;49(11):171925.

18. Buchs NC, Gervaz P, Secic M, Bucher P, Mugnier-Konrad $\mathrm{B}$, Morel P. Incidence, consequences, and risk factors for anastomotic dehiscence after colorectal surgery: a prospective monocentric study. Int J Colorectal Dis 2008;23(3):265-70.

19. Vignali A, Fazio VW, Lavery IC, Milsom JW, Church JM, Hull TL, et al. Factors associated with the occurrence of leaks in stapled rectal anastomoses: a review of 1,014 patients. J Am Coll Surg 1997;185(2):105-13.

20. Santos Jr JCM. Profilaxia das complicações pós-opertórias no tratamento cirúrgico das doenças do intestino grosso: II- Deiscência da anastomose. Rev Bras Coloproctol 1998;18(1):44-51.

21. Matthiessen $P$, Hallböök $\mathrm{O}$, Andersson $\mathrm{M}$, Rutegård $\mathrm{J}$, Sjödahl R. Risk factors for anastomotic leakage after anterior resection of the rectum. Colorectal Dis 2004;6(6):462-9.

22. Santos Jr JCM. Fatores de riscos associados às complicações cirúrgicas em operações de ressecções e anastomoses do intestino grosso sem o preparo mecânico: estudo da incidência de infecção e deiscência da anastomose. Rev Bras Coloproctol 2005;25(2):168-87.

23. Pronio A, Di Filippo A, Narilli P, Mancini B, Caporilli D, Piroli S, et al. Anastomotic dehiscence in colorectal surgery. Analysis of 1290 patients. Chir Ital 2007;59(5):599-609.

24. Fazio VW, Ziv Y, Church JM, Oakley JR, Lavery IC, Milsom $\mathrm{JW}$, et al. Ileal pouch-anal anastomoses complications and function in 1005 patients. Ann Surg 1995;222(2):120-7.

25. Dayton MT, Larsen KR, Christiansen DD. Similar functional results and complications after ileal pouch-anal anastomosis in patients with indeterminate vs ulcerative colitis. Arch Surg 2002;137(6):690-4; discussion 694-5.

26. Urbach DR, Kennedy ED, Cohen MM. Colon and rectal anastomoses do not require routine drainage: a systematic review and meta-analysis. Ann Surg 1999;229(2):174-80.

27. Biondo S, Parés D, Kreisler E, Ragué JM, Fraccalvieri D, Ruiz AG, et al. Anastomotic dehiscence after resection and primary anastomosis in left-sided colonic emergencies. Dis Colon Rectum 2005;48(12):2272-80.

28. Block JE, Moossa AR. Operative Colorectal Surgery. W.B. Saunders Company, 1994. pp. 622.

29. Bozzetti F. Pelvic drainage and other risk factors for leakage after anterior resection in rectal cancer patients. Ann Surg 2005;242(6):902.

30. Yeh CY, Changchien CR, Wang JY, Chen JS, Chen HH, Chiang JM, et al. Pelvic drainage and other risk factors for leakage after elective anterior resection in rectal cancer patients: a prospective study of 978 patients. Ann Surg 2005;241(1):9-13.

31. Komen N, Dijk JW, Lalmahomed Z, Klop K. After-hours colorectal surgery: a risk factor for anastomotic leakage. Int J Colorectal Dis 2009;24(7):789-95.

32. Marusch F, Koch A, Schmidt U, Geibetaler S, Dralle $\mathrm{H}$, Saeger HD, et al. Value of a protective stoma in low anterior resections for rectal cancer. Dis Colon Rectum 2002;45(9):1164-71.

33. Peeters KC, Tollenaar RA, Marijnen CA, Klein Kranenbarg E, Steup WH, Wiggers T, et al. Risk factors for anastomotic failure after total mesorectal excision of rectal cancer. Br J Surg 2005;92(2):211-6.

34. Nicksa GA, Dring RV, Johnson KH. Anastomotic leaks: what is the best diagnostic imaging study? Dis Colon Rectum 2007;50(2):197-203.

35. Johnson WC, Ulrich F, Mequid MM, Lepak N, Bowe $\mathrm{P}$, Harris $\mathrm{P}$, et al. Role of delayed hypersensitivity in predicting postoperative morbidity and mortality. Am J Surg 1979; 137(4):536-42.

36. Christou NV, Superina R, Broadhead M, Meakins JL. Postoperative depression of host resistance: determinants and effect of peripheral protein-sparing therapy. Surgery 1982;92(4):786-92.

37. Cheadle WG, Pemberton RM, Robinson D, Livingston DH, Rodriguez JL, Polk HC Jr. Lymphocyte subset responses to trauma and sepsis. J Trauma 1993;35(6):844-9.

38. Bone RC. Sir Isaac Newton, sepsis, SIRS, and CARS. Crit Care Med 1996;24(7):1125-8.

39. Bone RC, Balk RA, Cerra FB, Dellinger RP, Fein AM, Knaus WA, et al. Defintions for seppsis ando organ falilure and guidelines for the use of innovative therapies in sepsis. The ACCP/SCCM Consensus Conference Committee. American College of Chest Physicians/Society of Critical Care Medicine. Chest 1992;101:1644-55.

40. Dellinger RP, Levy MM, Carlet JM, Bion J, Parker MM, Jaeschke R, et al. Surviving Sepsis Campaign: international guidelines for management of severe sepsis and septic shock: 2008. Intensive Care Med 2008;34:17-60.

41. Santos Jr JCM, Levi CE. Tratamento das peritonites purulentas generalizadas usando como terapêutica coadjuvante a associação de clindamicina com gentamicina. Arq Bras Med 1987;61:355-60.

42. Santos Jr JCM, Levy CE, Reis CU. Comparative effectiveness of pefloxacin plus metronidazole and gentamicin plus metronidazole in the coadjuvant treatment of peritoneal infections. Clin Drug Investigation 1994;8(1):1-9.

43. Corman ML. Colon and Rectal Surgery, 5th Edition. USA: Lippincott Williams\&Wilkins; 2005. pp. 1695.

44. Pusajó JF, Bumaschny E, Doglio GR, Cherjovsky MR, Lipinszki AI, Hernández MS, et al. Postoperative intraabdominal sepsis requiring reoperation. Value of a predictive index. Arch Surg 1993;128(2):218-22; discussion 223.

45. Koperna T, Schulz F. Relaparotomy in peritonitis: prognosis and treatment of patients with persisting intraabdominal infection. World J Surg 2000;24(1):32-7.

46. Pitcher WD, Musher DM. Critical importance of early diagnosis and treatment of intra-abdominal infection. Arch Surg 1982;117(3):328-33.

47. Bohnen J, Boulanger M, Meakins JL, McLean AP. Prognosis in generalized peritonitis. Relation to cause and risk factors. 
Arch Surg 1983;118(3):285-90

48. Bone RC. Why sepsis trials fail. JAMA 1996;276(7):565-6.

49. Santos Jr JCM. Peritonite - infecção peritoneal e sepse. Rev Bras Coloproctol 2001;21(1):33-41.

50. Bosscha K, van Vroonhoven TJ, van der Werken C. Surgical management of severe secondary peritonitis. Br J Surg 1999;86(11):1371-7.

51. Mulier S, Penninckx F, Verwaest C, Filez L, Aerts R, Fieuws S, et al. Factors affecting mortality in generalized postoperative peritonitis: multivariate analysis in 96 patients. World J Surg 2003;27(4):379-84.

52. Lynch AC, Delaney CP, Senagore AJ, Connor JT, Remzi FH, Fazio VW. Clinical outcome and factors predictive of recurrence after enterocutaneous fistula surgery. Ann Surg 2004;240(5):825-31.

53. Rombeau JL, Fong J. What's new in treatment of colonic fistulae. Contemporary Surgery 2006;62(6):255-60.

54. Hwang TL, Chen MF. Short note: Randomized trial of fibrin tissue glue for low output enterocutaneous fistula. Br J Surg 1996;83:112.

55. Lamont JP, Hooker G, Espenschied JR, Lichliter WE, Franko E. Closure of proximal colorectal fistulas using fibrin sealant. Am Surg 2002;68:615-8.

56. Avalos-González J, Portilha-deBuen E, Leal-Cortés CA, Orozco-Mosqueda A, Estrada-Aguilar Mdel C, VelázquezRamírez GA, Reduction of the closure time of postoperative enterocutaneous fistulas with fibrin sealant. World J Gastroent 2010;16(22):2793-800.

57. Sciumè C, Geraci G, Piesello F, Li Volsi F, Facella T, Modica G. Infrequent early complication of mechanical digestive anastomosis: bleeding. Personal experience. Ann Ital Chir 2006;77(3):260-72.

58. Malik N, East JE, Buchanan GN, Kennedy RH. Endoscopic haemostasis of staple-line haemorrhage following colorectal resection. Colorectal Dis 2008;10(6):616-8.

59. Cirocco WC, Golub RW. Endoscopic treatment of postoperative hemorrhage from a stapled colorectal anastomosis. Am Surg 1995;61(5):460-3.

60. Perez RO, Souza AJ, Bresciani C, Proscurshim I, Coser R, Kiss D, et al. Endoscopic management of postoperative stapled colorectal anastomosis hemorrhage. Tech Coloproctol 2007;11(1):64-6.

61. Briel JW, Tamhankar AP, Hagen JA, DeMeester SR, Johansson J, Choustoulakis E, et al. Prevalence and risk factors for ischemia, leak, and stricture of esophageal anastomosis: Gastric pull-up versus colon interposition. J Am Coll Surg 2004;198:536-42.

62. Villanueva E, Sierra-Montenegro E, Rojas-Illanes M, Espartaza JPP, et al. Double stapler technique in colorectal surgery. Cir Ciruj 2008;78:49-53.

63. Baran JJ, Goldstein SD, Resnik AM. The double-staple technique in colorectal anastomoses: a critical review. Am Surg 1992;58(4):270-2.

64. Schlegel RD, Dehni N, Parc R, Caplin S, Tiret E. Results of reoperation in colorectal anastomotic strictures. Dis Colon Rectum 2001;44(10):1464-8.

65. Luchtefeld MA, Milsom JW, Senagore A, Surrell JA, Mazier WP. Colorectal anastomotic stenosis: Results of a survey of the ASCRS Membership. Dis Colon Rectum 1989;32(9):733-6.

66. Hood K, Lewis A. Dilator for high rectal strictures. Br J Surg 1986;73(8):633.

67. Lin SC, Sy E, Lin BW, Lee JC. Management of colorectal anastomotic strictures using multidiameter balloon dilation. J Soc Colon Rectal Surgeon (Taiwan) 2009;20:62-8.

68. Moraes RS, Buess G, Lima JHF, Morgenstern GA, Schulz GJ, Campos ACL, et al. Transanal endoscopic microsurgery (TEM) in the treatment of postoperative colorectal stenosis. Arq Bras Cir Dig 2008;21:147-9.

Endereço para correspondência: JÚLIO CÉSAR MONTEIRO SANTOS JR.

Avenida Min. Urbano Marcondes, 516 CEP 12515-230 - Guaratinguetá (SP), Brasil

E-mail: instmed@provale.com.br 